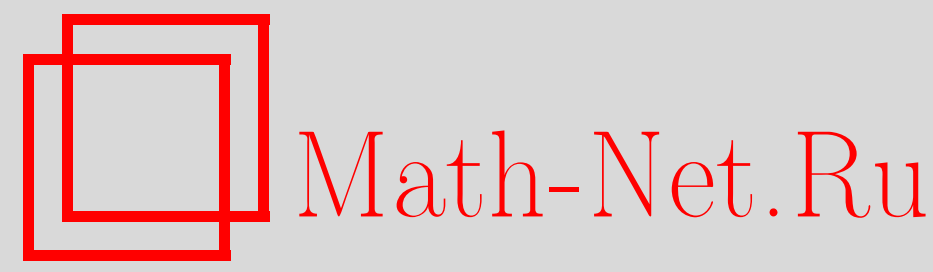

Е. М. Матвеев, О последовательных минимумах расширенной логарифмической высоты алгебраических чисел, Матем. сб., 1999, том 190, номер 3, 89-108

DOI: https://doi.org/10.4213/sm394

Использование Общероссийского математического портала Math-Net.Ru подразумевает, что вы прочитали и согласны с пользовательским соглашением http://www.mathnet.ru/rus/agreement

Параметры загрузки:

IP: 52.6 .47 .48

26 апреля 2023 г., $14: 53: 37$ 


\author{
Е. М. Матвеев
}

\title{
О последовательных минимумах расширенной логарифмической высоты алгебраических чисел
}

Пусть $\mathbb{K} \subseteq \mathbb{C}$ - алгебраическое поле; $S=2$, если $\mathbb{K}$ комплексное, и $S=1$, если $\mathbb{K} \subseteq \mathbb{R} ; \bar{\delta}=[\mathbb{K}: \mathbb{Q}] / S$. Для $\alpha \in \mathbb{K}^{*}$ положим $H_{*}(\alpha)=\max \{\delta h(\alpha),|\ln \alpha|\}$, где $h(\alpha)$ - высота Вейля числа $\alpha$. Тогда для мультипликативно независимых $\alpha_{1}, \ldots, \alpha_{n} \in \mathbb{K}^{*}$ вьполняется неравенство

$$
H_{*}\left(\alpha_{1}\right) \cdots H_{*}\left(\alpha_{n}\right) 2.5^{n}\left(e^{0.2 n} n\right)^{S} \delta \ln (4.64 \delta)>1
$$

Библиографиия: 19 названий.

Настоящая работа содержит описание математической конструкции, названной автором расширенньм логарифмическим пространством, и соответствующих мультипликативных алгебраических решеток, а также доказательства ряда неравенств для этих решеток.

План статьи следующий. В $\S \S 1-3$ приводятся общие сведения об алгебраических решетках. При этом в классическую конструкцию вносится небольшое усовершенствование, делаюшее ее более естественной и позволяющее уточнить известные неравенства (см. предложения 2.1-2.3). В $\S 4-5$ представлено описание расширенного логарифмического пространства и его простейших свойств. Основные результаты сформулированы в $\S 6$, а остаток статьи занимают доказательства этих теорем.

Выделим две важнейшие особенности полученных результатов. Во-первых, зависимость оценок от $D$ имеет вид $D \ln D(D-$ степень алгебраического поля). Во-вторых, зависимость от $n$ показательная и не содержит множителя $n^{n}(n-$ количество последовательных минимумов).

Основным методом настояшей работы является использование так называемых интерполяционных определителей, введенных недавно М. Лораном и заменяющих технику арифметико-аналитического продолжения Гельфонда. При этом не только доказательства становятся короче и прозрачнее, но и выводимые константы сушественно улучшаются. Это позволило получить явные оценки с достаточно мальми значениями входящих в них числовых констант.

Следует также отметить, что первоначально техника интерполяционного определителя и прием устранения множителя $n^{n}$ были использованы соответственно М. Лораном и автором в конструкциях значительно более сложных, чем в этой работе. Поэтому статья может быть полезна для знакомства с этими методами.

Работа имеет непосредственное приложение к теории линейных форм от логарифмов алгебраических чисел, а также может быть использована при конструировании новых типов многомерных решеток. 


\section{§1. Решетки}

Основные факты по геометрии чисел взяты из [1], [2] и [3].

Пусть дано нормированное векторное пространство $\mathscr{V}$ над $\mathbb{R}$. Тогда решеткой называется дискретная подгруппа $\Lambda \subseteq \mathscr{V}$. У решетки есть базис, все векторы решетки целочисленно выражаются через него. Количество векторов в базисе называется рангом решетки $\operatorname{rank} \Lambda$ (ввиду дискретности $\Lambda$ имеем: $\operatorname{rank}_{\mathbb{Z}} \Lambda=\operatorname{rank}_{\mathbb{R}} \Lambda$ ).

Для набора векторов $\mathscr{U}$ и множества $\mathscr{B} \subseteq \mathbb{R}$ через $\langle\mathscr{U}\rangle_{\mathscr{B}}$ будем обозначать множество линейных комбинаций векторов из $\mathscr{U}$ с коэффициентами из $\mathscr{B}$. Чтобы конечно порожденная аддитивная подгруппа $\Lambda$ являлась решеткой, должно выполняться условие: $\operatorname{rank} \Lambda=\operatorname{dim}\langle\Lambda\rangle_{\mathbb{R}}$. Если $\langle\Lambda\rangle_{\mathbb{R}}=\mathscr{V}$, то говорят, что решетка $n$ олная.

Скалярное произведение в векторном пространстве задает $m$-мерные объемы, индуцированные формулой

$$
\operatorname{Vol}_{m}\left\langle\mathbf{v}_{1}, \ldots, \mathbf{v}_{m}\right\rangle_{[0 ; 1)}=\left(\operatorname{det}\left(\mathbf{v}_{i}, \mathbf{v}_{j}\right)_{i, j=1, \ldots, m}\right)^{1 / 2},
$$

где $\left\langle\mathbf{v}_{1}, \ldots, \mathbf{v}_{m}\right\rangle_{[0 ; 1)}$ - параллелепипед, построенный на линейно независимых век-

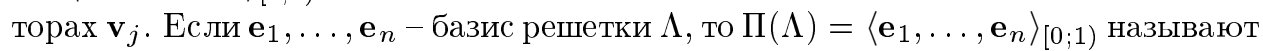
фундаментальным параллелепипедом решетки, а число $\operatorname{Vol}_{n} \Lambda=\operatorname{Vol}_{n} \Pi(\Lambda)$ называют объемом решетки $\Lambda$. Он не зависит от выбора базиса.

Если задана какая-либо норма $\|\cdot\|$, то через $\mathscr{W}(\|\cdot\|)$ будем обозначать единичный шар этой нормы, т.е. множество векторов $\mathbf{v}$ таких, что $\|\mathbf{v}\| \leqslant 1$.

Теперь напомним понятие последовательного минимума. Число $\lambda_{k}$ является $k$-м последовательным минимумом множества $\Lambda$ относительно нормирования $\|\cdot\|$, если $\lambda_{k}$ есть точная нижняя грань таких $\lambda$, для которых существуют $k$ линейно независимых векторов из $\lambda \mathscr{W}(\|\cdot\|) \cap \Lambda$. Отметим, что у решетки ввиду ее дискретности существуют ненулевые последовательные минимумы, достигающиеся на некоторых векторах. Последовательные минимумы не убывают, и их количество равно rank $\lambda$.

Минимальное расстояние в данной норме между различньми векторами решетки назовем ее диаметром $\operatorname{diam}(\Lambda,\|\cdot\|)=\lambda_{1}$. Это означает, что с помощью $\Lambda$ получается решетчатая упаковка пространства телами $\lambda_{1} \mathscr{W}(\|\cdot\|) / 2$ диаметра $\lambda_{1}$. Если норма не указана, то по умолчанию полагаем ее евклидовой и пишем $\operatorname{diam} \Lambda$.

Для $n$-мерной решетки $\Lambda$ можно ввести безразмерный параметр- иентральную плотность

$$
\gamma(\Lambda)=\frac{\operatorname{diam}^{n} \Lambda}{\operatorname{Vol}_{n} \Lambda}
$$

Содержательный смысл $\gamma(\Lambda)$ состоит в следующем. Если $\Lambda^{\prime}=\Lambda / \operatorname{diam} \Lambda$, то $\operatorname{diam} \Lambda^{\prime}=1$, а число $\gamma(\Lambda)=\gamma\left(\Lambda^{\prime}\right)$ равно среднему числу точек (центров) решетки $\Lambda^{\prime}$ в единице объема.

ПрЕДЛОЖЕНИЕ 1.1 (теорема Минковского о последовательных минимумах). Пусть в п-мерном евклидовом пространстве дань норма $\|\cdot\|$ и полная решетка $\Lambda$, ее последовательные минимумы $\lambda_{1}, \ldots, \lambda_{n}$ достигаются на векmорах $\mathbf{v}_{1}, \ldots, \mathbf{v}_{n}, \Lambda^{\prime}=\left\langle\mathbf{v}_{1}, \ldots, \mathbf{v}_{n}\right\rangle_{\mathbb{Z}},\left[\Lambda: \Lambda^{\prime}\right]-$ индекс $\Lambda$ относительно $\Lambda^{\prime}$. 
Тогда

$$
\frac{2^{n}}{n !}\left[\Lambda: \Lambda^{\prime}\right] \operatorname{Vol}_{n} \Lambda \leqslant \lambda_{1} \cdots \lambda_{n} \operatorname{Vol}_{n} \mathscr{W}(\|\cdot\|) \leqslant 2^{n} \operatorname{Vol}_{n} \Lambda
$$

\section{§2. Аддитивные алгебраические решетки}

Основные факты $\S \S 2$ и 3 взяты из [1], [3], хотя конструкция несколько отлична, на что обрашается специальное внимание.

Пусть дано алгебраическое поле $\mathbb{K}$ степени $[\mathbb{K}: \mathbb{Q}]=D$. Для каждого $\alpha \in \mathbb{K}$ имеется набор сопряженных чисел $\alpha^{(\sigma)} \in \mathbb{C}, \sigma=1, \ldots, D$. Это определяет гомоморфизм $g$ аддитивной группы $\mathbb{K}$ в $\mathbb{C}^{D} \cong \mathbb{R}^{2 D}$, задаваемый формулой $g(\alpha)=$ $\left(\alpha^{(1)}, \ldots, \alpha^{(D)}\right)$.

Зарезервируем обозначения: $\sigma=1, \ldots, D_{1}$ - гомоморфизмы поля $\mathbb{K}$ в $\mathbb{R} \subseteq \mathbb{C}$, а $\sigma=D_{1}+1, \ldots, D_{1}+2 D_{2}-$ комплексные гомоморфизмы, при этом $\sigma=D_{1}+t$ и $\tau=D_{1}+D_{2}+t\left(1 \leqslant t \leqslant D_{2}\right)$ соответствуют комплексно сопряженным вложениям $\mathbb{K}$ в $\mathbb{C}$. Для краткости будем говорить, что такие $\sigma$ и $\tau$ комплексно сопряжены $\left(D=D_{1}+2 D_{2}\right)$. Тогда наше геометрическое отображение обладает очевидным свойством: образ $g(\mathbb{K})$ лежит в $\mathbb{R}$-подпространстве $\mathscr{V}_{\text {а }} \subseteq \mathbb{C}^{D}$, которое задается условиями

$$
\begin{cases}\operatorname{Im} z_{\sigma}=0, & \text { если } \sigma-\text { действительный гомоморфизм, } \\ z_{\sigma}=\bar{z}_{\tau}, & \text { если } \sigma \text { и } \tau \text { комплексно сопряжены, }\end{cases}
$$

или, что эквивалентно,

$$
\begin{cases}y_{\sigma}=0, & \text { если } \sigma-\text { действительный гомоморфизм, } \\ x_{\sigma}=x_{\tau}, y_{\sigma}=-y_{\tau}, & \text { если } \sigma \text { и } \tau \text { комплексно сопряжены; }\end{cases}
$$

считаем, что координаты $z_{\sigma}=x_{\sigma}+i y_{\sigma}$ векторов $\mathbf{v} \in \mathbb{C}^{D}$ при стандартном овешествлении переходят в пару действительных координат $\left(x_{\sigma}, y_{\sigma}\right)$. Символ $i$ будет зарезервирован за мнимой единицей.

Зададим в $\mathbb{C}^{D}$ стандартную эрмитову форму

$$
(\mathbf{z}, \mathbf{w})=z_{1} \bar{w}_{1}+\cdots+z_{D} \bar{w}_{D}
$$

ПРЕДЛОЖЕНИЕ 2.1. На пространстве $\mathscr{V}$ а форма (2.2) является евклидовой.

ДокАЗАТЕЛЬСтво. Надо показать, что $(\mathbf{z}, \mathbf{w})$ принимает на $\mathscr{V}$ а вешественные значения. Для вешественных $\sigma$ очевидно $z_{\sigma} \bar{w}_{\sigma} \in \mathbb{R}$. Для комплексно сопряженных $\sigma, \tau$ имеем

$$
z_{\sigma} \bar{w}_{\sigma}+z_{\tau} \bar{w}_{\tau}=z_{\sigma} \bar{w}_{\sigma}+\bar{z}_{\sigma} w_{\sigma} \in \mathbb{R}
$$

откуда следует утверждение. 
ПРимечАния. 1. Стандартным геометрическим отображением $g_{0}$ (см. [1], [3]) считается аналогичное отображение, но в $\mathscr{V}_{0} \cong \mathbb{R}^{D_{1}} \times \mathbb{C}^{D_{2}}$ с отбрасыванием координат, связанных соотношениями (2.1). Фактически $\mathscr{V}_{0}$ можно считать координатным $\mathbb{R}$-подпространствомпространства $\mathbb{C}^{D}$ (одна из пары связанных координат равна нулю), а $g_{0}$ получается как композиция отображения $g$ и оператора проектирования $\mathscr{V}_{\text {a }}$ на $\mathscr{V}_{0}$.

2. В [4] поле $\mathbb{K}$, рассматриваемое как $\mathbb{Q}$-пространство, снабжено евклидовой нормой, аналогичной (2.2),

$$
\|\alpha\|^{2}=\left|\alpha^{(1)}\right|^{2}+\cdots+\left|\alpha^{(D)}\right|^{2}
$$

без построения соответствующего геометрического отображения. Очевидно, $g$ и есть нужное изометрическое вложение $\mathbb{K}$ в геометрическое пространство.

ПРЕДЛОЖЕНИЕ 2.2 (см. [3]). Пусть $\mathbb{Z}_{\mathbb{K}}-$ кольцо иельх чисел поля $\mathbb{K}$, $\operatorname{Disc} \mathbb{K}$ - дискриминант $\mathbb{K}$. Тогда $g_{0}\left(\mathbb{Z}_{\mathbb{K}}\right)$ - полная решетка в $\mathscr{V}_{0}$ обгема $2^{-D_{2}}|\operatorname{Disc} \mathbb{K}|^{1 / 2}$ и диаметра $\lambda_{1}$,

$$
\left(\frac{D_{1}}{2}+D_{2}\right)^{1 / 2} \leqslant \lambda_{1} \leqslant\left(D_{1}+D_{2}\right)^{1 / 2}
$$

Таким образом, отображение $g_{0}$ удобно тем, что образ $g_{0}\left(\mathbb{Z}_{\mathbb{K}}\right)$ полон в $\mathscr{V}_{0}$. Покажем, что в случае отображения $g$ формулы для объема и диаметра оказываются проше, а в случае $g_{0}$ эти формулы усложняются из-за действия проекции $\mathscr{V}_{\mathrm{a}}$ на $\mathscr{V}_{0}$.

ПРЕДЛОЖЕНИЕ 2.3. $\Lambda_{\mathrm{a}}=g\left(\mathbb{Z}_{\mathbb{K}}\right)$ - полная решетка в $\mathscr{V}_{\mathrm{a}}$ обгема $|\operatorname{Disc} \mathbb{K}|^{1 / 2}$ u диаметра $\lambda_{1}=D^{1 / 2}$.

ДокАЗАТЕЛЬство. Утверждение о полноте и объеме следует из того, что проектирование $\mathscr{V}_{\text {а }}$ на $\mathscr{V}_{0}$ - это изоморфизм, уменьшающий $D$-мерные объемы в $2^{D_{2}}$ раз (отбрасывание одной из двух связанных координат уменњшает объем в $\sqrt{2}$ раз, число связанных пар $\mathbb{R}$-координат равно $2 D_{2}$ ). Оценка диаметра вытекает из соотношения между средним арифметическим и средним геометрическим

$$
\frac{|g(\alpha)|^{2}}{D} \geqslant\left(\left|\alpha^{(1)}\right|^{2} \cdots\left|\alpha^{(D)}\right|^{2}\right)^{1 / D}=\left|\operatorname{Norm}_{\mathbb{K}} \alpha\right|^{2 / D} \geqslant 1
$$

Равенство достигается для $\alpha=1$, для всех корней из 1 и только для них.

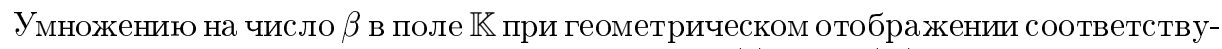
ет линейный оператор $\mathscr{B}$ в $\mathbb{C}^{D}$ с матрищей $\operatorname{diag}\left(\beta^{(1)}, \ldots, \beta^{(D)}\right)$. Очевидно, $\mathscr{V}_{\text {a }}$ является инвариантным $\mathbb{R}$-подпространством для $\mathscr{B}$. Укажем еще одно инвариантное $\mathbb{R}$-подпространство. В $\mathbb{C}$ умножению на $i$ соответствует поворот на угол $\pi / 2$. Поэтому $i \mathscr{V}_{\text {а }}$ будет ортогональньгм к $\mathscr{V}_{\text {а }}$ и инвариантньгм для $\mathscr{B}$ (ввиду равенства $i \mathscr{B}=\mathscr{B} i)$. Таким образом, при овешествлении $\mathbb{C}^{D}$ оператор $\mathscr{B}$ распадается на прямую сумму двух подобных операторов. 


\section{§3. Логарифмическое пространство}

При изучении мультипликативной группы $\mathbb{K}^{*}$ мы всегда будем предполагать, что $\alpha \neq 0$, не оговаривая это особо.

Отображение $\mathbb{K}^{*}$ в $\mathbb{R}^{D}$, задаваемое формулами

$$
x_{\sigma}=x_{\sigma}(\alpha)=\ln \left|\alpha^{(\sigma)}\right|, \quad 1 \leqslant \sigma \leqslant D,
$$

является гомоморфизмом мультипликативной группы поля $\mathbb{K}$. Как и в аддитивном случае, для комплексно сопряженных $\sigma$ и $\tau$ выполняется $x_{\sigma}=x_{\tau}$. Однако здесь образ $\mathbb{K}^{*}$ не является решеткой из-за нарушения дискретности. Решеткой (обозначим ее через $\Lambda_{U}$ ) будет только образ групшы $U(\mathbb{K})$ единиц поля $\mathbb{K}$. Ранг $\Lambda_{U}$ согласно теореме Дирихле равен $r=D_{1}+D_{2}-1$, поскольку для $\alpha \in U(\mathbb{K})$ выполняется еше одно соотношение

$$
x_{1}(\alpha)+\cdots+x_{D}(\alpha)=\ln \left|\operatorname{Norm}_{\mathbb{K}} \alpha\right|=0
$$

При погружении этой решетки в $r$-мерное координатное пространство обычно (см. [1]) пары координат $x_{\sigma}=x_{\tau}$ заменяют на $2 x_{\sigma}$, что сохраняет соотношение типа (3.1), но увеличивает объемы в $\sqrt{2}$ раз. Затем одну (любую) из оставшихся $r+1$ координат отбрасьвают. Это уменњшает объем в $\sqrt{r+1}$ раз. Объем $R(\mathbb{K})$ полученной решетки называется регулятором поля $\mathbb{K}$. Ясно, что объем исходной решетки $\Lambda_{U}$ в этом случае равен $\operatorname{Vol}_{r} \Lambda_{U}=\left(2^{-D_{2}}(r+1)\right)^{1 / 2} R(\mathbb{K})$.

Для более детального исследования мультипликативной структуры поля $\mathbb{K}$ часто применяется следующая конструкция. Положим $\mathscr{D}=\{1, \ldots, D\}$. Пронумеруем простые идеалы поля $\mathbb{K}$ индексами $\sigma>D$ в порядке возрастания их норм и зададим нормирования $\mathbb{K}$ по формуле

$$
|\alpha|_{\sigma}= \begin{cases}\left|\alpha^{(\sigma)}\right|, & 1 \leqslant \sigma \leqslant D \\ (\operatorname{Norm} \mathfrak{p})^{-\operatorname{ord}_{\mathfrak{p}} \alpha}, & \mathfrak{p}=\mathfrak{p}_{\sigma}, \quad \sigma>D\end{cases}
$$

Мы будем учитывать все такие нормирования несмотря на то, что они одинаковы для комплексно сопряженных $\sigma$. Это позволяет записывать формулу произведения без весовых коэффициентов

$$
\prod_{\sigma=1}^{\infty}|\alpha|_{\sigma}=1, \quad \alpha \in \mathbb{K}^{*}
$$

Также отметим, что только для конечного числа нормирований $|\alpha|_{\sigma}$ отличны от единицы.

Рассмотрим логарифмическое отображение $\mathscr{L}$ мультипликативной группы $\mathbb{K}^{*}$ в бесконечномерное пространство $\mathbb{R}^{\infty}$ векторов $\mathbf{x}=\left(x_{\sigma} \in \mathbb{R}: 1 \leqslant \sigma<\infty\right)$, задаваемое формулой

$$
x_{\sigma}=x_{\sigma}(\alpha)=\ln |\alpha|_{\sigma} \quad(1 \leqslant \sigma<\infty)
$$

Очевидно, это гомоморфизм, а образ $\Lambda_{\mathrm{m}}=\mathscr{L}\left(\mathbb{K}^{*}\right)$ лежит в подпространстве $\mathscr{E} \subseteq \mathbb{R}^{\infty}$, определяемом условиями: $\mathbf{x} \in \mathscr{E}$, если 
(a) почти все $x_{\sigma}=0$;

(b) $x_{\sigma}=x_{\tau}$ для комплексно сопряженных $\sigma$ и $\tau$;

(c) выполняется аддитивный аналог формулы произведения

$$
\sum_{\sigma=1}^{\infty} x_{\sigma}=0
$$

(ср. с (3.1)). Будем называть $\mathscr{E}$ логарифмическим пространством.

Отметим, что для логарифмических образов $\mathbf{x}(\alpha)$ выполняется

$$
x_{\sigma} \in \ln \left(\operatorname{Norm} \mathfrak{p}_{\sigma}\right) \mathbb{Z}, \quad \sigma>D
$$

Введем в пространстве $\mathscr{E}$ нормирование

$$
H(\mathbf{x})=\frac{1}{2} \sum_{\sigma=1}^{\infty}\left|x_{\sigma}\right|=\sum_{\sigma=1}^{\infty} \max \left\{0, x_{\sigma}\right\}
$$

Если $\mathbf{x}=\mathscr{L}(\alpha)$, то говорят, что $H(\alpha)=H(\mathbf{x})$ - это логарифмическая высота $\alpha$. Следует отметить, что логарифмическая высота зависит от $\mathbb{K}$, т.е. $H(\alpha)=$ $H(\alpha, \mathbb{K})$, хотя $\alpha$ можно помещать в различные поля. Однако при надлежащей нормализации можно получить инвариантное определение, а именно, норма $h(\alpha)=$ $H(\alpha) / D$, называемая абсолютной логарифмической высотой, не зависит от выбора поля, содержащего $\alpha$ (см. [5]). Если поле не фиксировано, то под $H(\alpha)$ будем понимать высоту в поле $\mathbb{Q}(\alpha)$.

По теореме Кронекера ядром логарифмического отображения является $U_{1}(\mathbb{K})$ - группа корней из 1 поля $\mathbb{K} ;$ эта группа конечна, циклична; $\Lambda_{\mathrm{m}}=\mathscr{L}\left(\mathbb{K}^{*}\right)$ является решеткой в $\mathscr{E}$.

Следствием теоремы Кронекера является то, что логарифмическое отображение необратимо, т.е. мы можем восстановить число $\alpha$ по его образу $\mathscr{L}(\alpha)$ только с точностью до множителя - корня из 1.

Уточненный вариант теоремы Дирихле о единицах звучит так.

ПРЕДЛОЖЕНИЕ 3.1. Пусть фиксирован конечный набор $\mathscr{S}$ индексов $\sigma$, $\mathscr{D} \subseteq \mathscr{S} . \quad$ Тогда множество чисел $U(\mathbb{K}, \mathscr{S})=\left\{\alpha \in \mathbb{K}^{*}:|\alpha|_{\sigma}=1, \sigma \notin \mathscr{S}\right\}$ (так называемые $\mathscr{S}$-единиць) образует при логарифмическом отображсении полную решетку ранга $r(\mathscr{S})=|\mathscr{S}|-D_{2}-1$ в подпространстве $\mathscr{E}_{0}(\mathscr{S})$ логарифмического пространства, заданном условиями $x_{\sigma}=0, \sigma \notin \mathscr{S}$.

Докажем простой, но важный результат об $\mathscr{L}\left(\mathbb{K}^{*}\right)$.

ЛЕмма 3.2. Для последовательных минимумов решетки $\mathscr{L}\left(\mathbb{K}^{*}\right)$ имеем

$$
\lambda_{n} \geqslant \ln \left(\operatorname{Norm} \mathfrak{p}_{n+D_{2}+1}\right), \quad n>r=D_{1}+D_{2}-1
$$


ДокАЗАТЕЛЬСтво. С учетом свойства (b) логарифмического пространства среди первых $D$ координат векторов $\mathbf{x} \in \mathscr{E}$ имеется $D_{2}$ одинаковых, что дает $D_{2}$ независимых линейных соотношений. Еще одно соотношение - это (3.3), очевидно, независимое от предыдущих. Поэтому если мы имеем $n \geqslant D-D_{2}$ линейно независимых векторов, то по крайней мере у одного из них должна быть ненулевая координата $\tau>n+D_{2}$. Но тогда для этой координаты $\left|x_{\tau}\right| \geqslant(1 / 2) \ln \left(\right.$ Norm $\left.\mathfrak{p}_{\tau}\right)$. Кроме того, из (3.3) следует, что сумма координат, противоположных по знаку $x_{\tau}$, также по модулю не меньше $(1 / 2) \ln \left(\mathrm{Normp}_{\tau}\right)$. Отсюда с учетом порядка нумерации идеалов следует утверждение леммы.

Вопрос о последовательных минимумах при $n<D-D_{2}$ изучен далеко не полностью. По сути дела, все результаты относятся только к $\lambda_{1}$, а для следующих $\lambda_{k}$ нетривиальных нижних оценок нет. Относительно $\lambda_{1}$ имеется недоказанная гипотеза Лемера [6] о том, что существует абсолютная константа $C>0$, для которой всегда выполняется $\lambda_{1} \geqslant C$. Найденное Лемером число $\theta_{10}=1.176 \ldots$, удовлетворяющее уравнению

$$
x^{10}+x^{9}-x^{7}-x^{6}-x^{5}-x^{4}-x^{3}+x+1=0
$$

имеет минимальную известную на сегодня логарифмическую высоту $H\left(\theta_{10}\right)=$ $\ln \left(\theta_{10}\right)>0$.

Добровольскому [7] принадлежит наилучшая асимптотическая нижняя оценка $\lambda_{1}$, константа в которой неоднократно уточнялась:

$$
H(\alpha) \gg\left(\frac{\ln \ln D}{\ln D}\right)^{3}, \quad \alpha \notin U_{1}(\mathbb{A}) .
$$

Здесь $U_{1}(\mathbb{A})$ - группа корней из 1 поля всех алгебраических чисел $\mathbb{A}$.

В [8] доказано, что в (3.5) с некоторыми оговорками можно заменить $D=\operatorname{deg} \alpha$ на меньшую величину $\delta=D /|\operatorname{Disc} \alpha|^{1 / D}$, при этом $\delta^{D / 2}$ - это центральная плотность решетки $g(\mathbb{Z}[\alpha])$. Таким образом, центральная плотность аддитивной решетки ограничивает снизу диаметр мультипликативной решетки.

Ввиду теоретической и прикладной важности вопроса интерес представляют также и явные оценки, пусть даже и худшей асимптотики, но в достаточно большом диапазоне значений $D$ превосходящие хорошие асимптотические оценки. Для логарифмической высоты здесь следует указать результат Бланскби и Монтгомери [9]:

$$
H(\alpha) \geqslant \ln \left(1+\frac{1}{52 D \ln D}\right), \quad \alpha \notin U_{1}(\mathbb{A}) .
$$

Кроме того, для широких классов алгебраических чисел имеются явные неулучшаемые оценки, соответствующие гипотезе Лемера, часть из которых мы резюмируем в виде утверждения.

ПРЕДЛОЖЕНИЕ 3.3. 1) Очевидно, если $\alpha \notin U(\mathbb{A}), m o H(\alpha) \geqslant \ln 2$.

2) Для невзаимных $\alpha$ ( $\alpha$ называется взаимным или возвратныцм, если $\alpha$ алгебраически сопряжено с $1 / \alpha$ или, эквивалентно, минимальный для $\alpha$ многочлен 
симметричен) выполняется $H(\alpha) \geqslant \ln \theta_{3}$, где $\theta_{3}=1.324 \ldots$ - корень уравнения $x^{3}-x-1=0$ (см. [10]).

3) Для цельх вполне вещественных $\alpha\left(\right.$ m.е. все $\left.\alpha^{(\sigma)} \in \mathbb{R}\right)$ выполняется

$$
H(\alpha) \geqslant \frac{D}{2} \ln \left(\frac{1+\sqrt{5}}{2}\right),
$$

(см. [11]).

4) Для иельх вполне полохсительных $\alpha$ выполняется

$$
H(\alpha) \geqslant D \ln \left(\frac{1+\sqrt{5}}{2}\right) .
$$

(Получается, если предыдуший результат применить $\mathrm{k} \sqrt{\alpha}$.)

В работе Бойда [12] перечислены все алгебраические числа малой логарифмической высоты степени $D \leqslant 20$.

Следует также отметить, что имеется значительное количество публикаций о регуляторе поля, связанном по теореме Минковского с произведением всех последовательных минимумов решетки единиц поля. Например, в работе Циммерта [13] показано, что $R(\mathbb{K})$ имеет как минимум показательньй рост в зависимости от $D$. В работе Фридмана [14] изучается рост $R(\mathbb{K})$ в зависимости от $|\operatorname{Disc} \mathbb{K}|$, т.е. от объема аддитивной решетки поля. Фактически же в этой зависимости участвует центральная плотность аддитивной решетки.

\section{§4. Расширенное логарифмическое пространство}

В настоящей работе рассматривается более сложная конструкция, чем приведенная вьше. Эта конструкция до сих пор не привлекала внимания исследователей (она отсутствует в недавнем обзоре [3]), но необходима и неявно используется в ряде вопросов, например при изучении линейных форм от логарифмов алгебраических чисел и всех задач с ними связанных (см. [15]). Сама конструкция с оценкой $\lambda_{1}$ была тезисно приведена в [16]. Дальнейшие результаты о $\lambda_{1}$ были получены в [17]. В нашей работе получены нетривиальные оценки $\lambda_{n}, n>1$. Схема доказательства та же, что и в [17].

Прежде всего построим конструкцию, которую назовем расширенным логарифмическим пространством. Сначала выберем некоторьй непустой набор индексов $\mathscr{S} \subseteq \mathscr{D}$ и заменим в пространстве $\mathbb{R}^{\infty}$, содержашем $\mathscr{E}, \mathbb{R}$-компоненты, соответствующие координатам $\sigma \in \mathscr{S}$, на $\mathbb{C}$-компоненты. Новая координата будет иметь вид $z_{\sigma}=x_{\sigma}+i y_{\sigma}$. Комплексно сопряженные $\sigma, \tau$ должны включаться в $\mathscr{S}$ парами. Для координат $x_{\sigma}$ сохраняются прежние условия (a)-(c) логарифмического пространства и добавляется еше одно для $y_{\sigma}$ :

(d) $y_{\sigma}=-y_{\tau}$ для комплексно сопряженных $\sigma$ и $\tau$.

ЗАмЕчАниЕ. В приложениях (см., например, [4]) условие (d) выполняется автоматически. Если же мы изучаем решетки с геометрической точки зрения, например ишем плотные упаковки шаров, то условие (d) можно отбросить. На результатах статьи это практически не отразится. 
Зарезервируем также для указанной конструкции обозначения: $\mathscr{S}$ содержит $s_{1}$ действительных координат и $s_{2}$ пар комплексно сопряженных координат, $S=$ $|\mathscr{S}|=s_{1}+2 s_{2}, s=s_{1}+s_{2}$.

Полученное пространство назовем расширенным логарифмическим пространством $\mathscr{E}(\mathscr{S})$. Можно считать, что $\mathscr{E} \subseteq \mathscr{E}(\mathscr{S})$, относя к $\mathscr{E}$ векторы, имеющие $y_{\sigma}=0$. Сушествует естественное отображение $\mathscr{E}(\mathscr{S})$ в $\mathscr{E}-$ проектирование $\mathscr{P}$, обращающее в нуль все $y_{\sigma}$.

Теперь опишем связь $\mathbb{K}^{*}$ с $\mathscr{E}(\mathscr{S})$. Для этого с координатами $x_{\sigma}$ поступим так же, как и в случае построения $\mathscr{E}$ по формуле (3.2). Координаты $y_{\sigma}$ определим как $y_{\sigma}=\operatorname{Arg} \alpha^{(\sigma)}$. Другими словами, $z_{\sigma}=\operatorname{Ln} \alpha^{(\sigma)}, \sigma \in \mathscr{S}$. При этом, строя соответствуюшую решетку в $\mathscr{E}(\mathscr{S})$, будем учитывать все возможные значения логарифма. Полученное множество точек в $\mathscr{E}(\mathscr{S})$ обозначим через $\Lambda(\mathscr{S})$. Отметим его основное свойство: для $\mathbf{x} \in \Lambda(\mathscr{S})$ при некотором $\alpha \in \mathbb{K}^{*}$ выполняется

$$
\begin{cases}\exp \left(x_{\sigma}\right)=|\alpha|_{\sigma}, & \sigma \notin \mathscr{S}, \\ \exp \left(z_{\sigma}\right)=\alpha^{(\sigma)}, & \sigma \in \mathscr{S}\end{cases}
$$

что позволяет восстанавливать $\alpha$ по его образу. Таким образом, расширенное логарифмическое отображение неоднозначно, зато существует сюръективное однозначное обратное отображение $\Lambda(\mathscr{S})$ на $\mathbb{K}^{*}, \alpha=\mathscr{A}(\mathbf{x}), \mathbf{x} \in \Lambda(\mathscr{S})$, определяемое по формулам (4.1).

Далее мы часто будем фиксировать некоторьй набор индексов $\mathscr{T}(\mathscr{D} \subseteq \mathscr{T})$, тогда $\mathscr{E}(\mathscr{S}, \mathscr{T})$ будет обозначать координатное подпространство пространства $\mathscr{E}(\mathscr{S})$, содержащее векторы с $x_{\sigma}=0, z_{\sigma}=0, \sigma \notin \mathscr{T}$. Через $\Lambda(\mathscr{S}, \mathscr{T})$ будем обозначать пересечение $\mathscr{E}(\mathscr{S}, \mathscr{T}) \cap \Lambda(\mathscr{S})$.

Сформулируем сначала простые свойства множества $\Lambda(\mathscr{S})$ и отображений $\mathscr{A}$ (восстановление чисел по их расширенным логарифмическим изображениям), $\mathscr{L}$ (логарифмическое отображение $\mathbb{K}^{*}$ в $\left.\mathscr{E} \subseteq \mathscr{E}(\mathscr{S})\right), \mathscr{P}$ (проектирование $\mathscr{E}(\mathscr{S})$ на $\mathscr{E}$, т.е. обнуление всех $\left.y_{\sigma}\right)$.

Лемма 4.1. Выполняются следующие свойства:

1) отображсение $\mathscr{A}$ - гомоморфизм $\Lambda(\mathscr{S})$ в $\mathbb{K}^{*}$, m.е.

$$
\mathscr{A}(\mathbf{u}+\mathbf{v})=\mathscr{A}(\mathbf{u}) \cdot \mathscr{A}(\mathbf{v})
$$

2) $\operatorname{Ker} \mathscr{A}$ состоит из векторов, у которых все $x_{\sigma}=0(1 \leqslant \sigma<\infty)$, а $y_{\sigma} \in 2 \pi \mathbb{Z}, \sigma \in \mathscr{S} ; \operatorname{rank} \operatorname{Ker} \mathscr{A}=s=s_{1}+s_{2} ;$

3) на множестве $\Lambda(\mathscr{S})$ выполняется равенство $\mathscr{P}=\mathscr{L} \mathscr{A}$;

4) $\mathscr{A}(\Lambda(\mathscr{S}) \cap \operatorname{Ker} \mathscr{P})=U_{1}(\mathbb{K})$;

5) $\operatorname{rank}_{\mathbb{Z}} \Lambda(\mathscr{S}, \mathscr{T}) \geqslant|\mathscr{T}|+s-D_{2}-1$.

ДокАЗАТЕЛЬСтво. Свойства 1-4 очевидны.

Для доказательства п. 5 отметим, что согласно теореме Дирихле существует $|\mathscr{T}|-D_{2}-1$ мультипликативно независимых $\mathscr{T}$-единиц. Если взять какие-либо их $\mathscr{A}$-прообразы в $\Lambda(\mathscr{S})$, то это будут линейно независимые векторы в $\Lambda(\mathscr{S}, \mathscr{T})$. 
Более того, они будут не сравнимы по $\bmod (\operatorname{Ker} \mathscr{A})$. Согласно п. 2 мы можем взять $s$ линейно независимых векторов из $\operatorname{Ker} \mathscr{A}$, ввиду вложения $\mathscr{D} \subseteq \mathscr{T}$ принадлежащих тому же множеству $\Lambda(\mathscr{S}, \mathscr{T})$ и при этом линейно независимых с предыдущими векторами. Этим п. 5 доказан.

Стандартное скалярное произведение позволяет говорить только о конечномерных объемах. Кроме того, оба пространства $\mathscr{E}$ и $\mathscr{E}(\mathscr{S})$ не являются гильбертовыми, так как состоят из векторов, у которых почти все координаты равны нулю, значит, нельзя говорить о метрической замкнутости и пр. Так что подобные вопросы в работе не затрагиваются. Кроме того, фактически в приложениях всегда берется какое-либо конечномерное координатное подпространство $\mathscr{E}(\mathscr{S}, \mathscr{T})$.

Как и в пространстве $\mathscr{E}$, говоря о решетках в $\mathscr{E}(\mathscr{S})$, мы будем опираться не на евклидову норму (нужную для определения объемов), а на норму, аналогичную норме (3.4) в $\mathscr{E}$. Для этого положим с $\theta>0$ :

$$
\begin{gathered}
\mu(\mathbf{x}, \mathscr{S})=\frac{1}{2 S} \sum_{\sigma \notin \mathscr{S}}\left|x_{\sigma}\right|, \\
\lambda(\mathbf{x}, \mathscr{S})=\frac{1}{S} \sum_{\sigma \in \mathscr{S}}\left|z_{\sigma}\right|, \quad H_{\theta}(\mathbf{x}, \mathscr{S})=\max \{\mu(\mathbf{x}, \mathscr{S}) \theta, \lambda(\mathbf{x}, \mathscr{S})\}, \\
\lambda^{*}(\mathbf{x}, \mathscr{S})=\max \left\{\left|z_{\sigma}\right|: \sigma \in \mathscr{S}\right\}, \quad H_{\theta}^{*}(\mathbf{x}, \mathscr{S})=\max \left\{\mu(\mathbf{x}, \mathscr{S}) \theta, \lambda^{*}(\mathbf{x}, \mathscr{S})\right\}
\end{gathered}
$$

и назовем $H_{\theta}(\mathbf{x}, \mathscr{S})$ расширенной логарифмической высотой (с весовьм коэффициентом $\theta$, определяющим соотношение между $\lambda$ и $\mu$ ). Если $\mathscr{A}(\mathbf{x})=\alpha$, то в случаях, когда нет опасности ошибиться, мы будем писать $\lambda(\alpha, \mathscr{S})=\lambda(\mathbf{x}, \mathscr{S})$ и пр., подразумевая, что в $\lambda$ выбраны некоторые значения логарифмов.

До сих пор в приложениях (см. [15]) в основном встречалась огрубленная форма расширенной логарифмической высоты

$$
H_{*}(\alpha)=\max \left\{\frac{D}{S} h(\alpha),|\ln \alpha|\right\}
$$

для случаев, когда взято либо только одно действительное $\sigma$ (если $S=1$ ), либо пара комплексно сопряженных $\sigma, \tau$ (если $S=2$ ), где $\lambda(\alpha, \mathscr{S})=|\ln \alpha|$, а $(D / S) h(\alpha) \geqslant \mu(\alpha, \mathscr{S})$. Возможны и другие аналогичные нормирования.

\section{§5. Сведения о первом последовательном минимуме}

Для работы с корнями из 1 нам потребуется следующее утверждение.

ПреДЛОЖЕНИЕ 5.1 (см., например, [15]). Пусть $\alpha=\exp (2 \pi i / N), \operatorname{mozда~} D=$

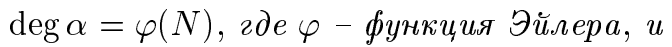

$$
|\ln \alpha| \geqslant \frac{\pi}{2 D \ln \ln (6 D)} \geqslant \frac{\pi}{2 D \ln (2 D)} .
$$

Отметим также, что если $\mathbb{K}$ не вполне комплексное поле, то в нем нет других корней из 1 , кроме $\alpha= \pm 1$.

Прежде чем переходить к основной теореме о первых последовательных минимумах $\Lambda(\mathscr{S})$, приведем простое утверждение, по сути означаюшее, что мы действительно имеем дело с полной решеткой. 
Лемма 5.2. 1) Для любого набора индексов $\mathscr{T}(\mathscr{D} \subseteq \mathscr{T})$ решетка $\Lambda(\mathscr{S}, \mathscr{T})$ является полной в $\mathscr{E}(\mathscr{S}, \mathscr{T})$ ранга $|\mathscr{T}|+s-D_{2}-1$.

2) Последовательные минимумы $\Lambda(\mathscr{S})$ относительно нормы $H_{\theta}(\cdot, \mathscr{S})$ удовлетворяют условию

$$
\lambda_{n} \geqslant \ln \left(\operatorname{Norm} \mathfrak{p}_{n+D_{2}-s+1}\right) \frac{2 \theta}{S \min \{4,2+\theta\}}, \quad n>r+s
$$

3) $\Lambda(\mathscr{S})$ - полная решетка в $\mathscr{E}(\mathscr{S})$.

ДоказАТЕЛЬСтво. Пусть $\mathbf{x} \in \Lambda(\mathscr{S}, \mathscr{T}), \mathbf{y}=\mathscr{P}(\mathbf{x}) \in \mathscr{E}$. Если $\mathbf{y} \neq \mathbf{0}$, то из $(4.2)$ и (3.4) получаем, что

$$
H_{\theta}(\mathbf{x}, \mathscr{S}) \geqslant \frac{\mu(\mathbf{x}, \mathscr{S}) 2 \theta+\lambda(\mathbf{x}, \mathscr{S}) \theta}{2+\theta} \geqslant H(\mathbf{y}) \frac{2 \theta}{(2+\theta) S}
$$

и по теореме Кронекера $H_{\theta}(\mathbf{x}, \mathscr{S})$ ограничена снизу константой. Если же $\mathbf{y}=\mathbf{0}$, то $\mathbf{x}$ является прообразом корня из 1 и согласно предложению 5.1 высота $H_{\theta}(\mathbf{x}, \mathscr{S})$ также ограничена снизу. Так что группа $\Lambda(\mathscr{S}, \mathscr{T})$ дискретна, т.е. является решеткой, и по лемме 4.1 ее ранг максимален.

Пункт 2 доказывается аналогично лемме 3.2 применением (5.2) или неравенства $H_{\theta}(\mathbf{x}, \mathscr{S}) \geqslant \mu(\mathbf{x}, \mathscr{S}) \theta$ с учетом того, что добавлено $S$ координат $y_{\sigma}$, из которых $s_{2}$ пар связаны. Поэтому номера $p$-адических нормирований сдвинуты на $s$ вперед.

Из первых двух пунктов следует, что $\Lambda(\mathscr{S})$ - решетка, при этом полнота получается за счет того, что $\mathscr{E}(\mathscr{S})$ порождается векторами, у которых почти все координаты равны нулю.

ПРЕДЛОЖЕНИЕ 5.3 (см. [17]). Пусть $\alpha \in \mathbb{K}^{*}, \eta \geqslant 0$ и выполняются условия

$$
\mu(\alpha, \mathscr{S}) \leqslant \frac{2 \eta}{(2+\eta) \xi}, \quad \lambda(\alpha, \mathscr{S}) \leqslant \frac{1}{(2+\eta) \xi}
$$

Тогда $\alpha=1$. Здесь $\xi=\delta \ln (4.8 \delta), \delta=D / S$.

Положив здесь $\eta=1 /(2 \theta)$, получим:

СлЕДСТВИЕ 5.4. При $\alpha \in \mathbb{K}^{*}, \alpha \neq 1, \delta=D / S$ выполняется

$$
H_{\theta}(\alpha, \mathscr{S})>\frac{2 \theta}{(1+4 \theta) \delta \ln (4.8 \delta)}
$$

Сравнение с предложением 5.1 показывает, что тип зависимости от $\delta(\delta \ln \delta$ в знаменателе) в оценках в основном правильный, и дальнейшие улучшения могут состоять только в замене $\ln \delta$ на $\ln \ln \delta$ и в уточнении параметров, не зависящих от $\delta$. Этим ситуация в логарифмическом пространстве отличается от положения в расширенном логарифмическом пространстве, где аналог гипотезы Лемера неверен. 
ПРЕДЛОЖЕНИЕ 5.5 (см. [17]). Для $\alpha \in \mathbb{K}^{*}, \alpha \neq 1$, выполняется

$$
\operatorname{sh} \frac{\lambda(\alpha, \mathscr{S})}{2} \geqslant \exp \left(-\frac{D}{S} \ln 2-\mu(\alpha, \mathscr{S})\right) .
$$

В [17] не было приведено полезное следствие из этой теоремы, уточняющее результат Шинцеля (см. предложение 3.3$){ }^{1}$

СлЕДСТВИЕ 5.6. Пусть $\alpha \neq 1$ - челое вполне положительное алгебраическое число. Тогда

$$
h(\alpha) \geqslant \ln \frac{1+\sqrt{1+4 y^{2}}}{2}, \quad y=|\operatorname{Norm} \alpha|^{1 / \operatorname{deg} \alpha} .
$$

ДокАЗАтельство. Положим $\mathbb{K}=\mathbb{Q}(\alpha), \mathscr{S}=\mathscr{D}=\{1, \ldots, D\}, S=D$. Поскольку $\alpha$ - целое число, то для неархимедовых нормирований имеем $|\alpha|_{\sigma} \leqslant 1$, $\sigma>D$. С учетом формулы произведения это дает

$$
\mu(\alpha, \mathscr{D})=-\frac{1}{2 D} \sum_{\sigma>D} \ln |\alpha|_{\sigma}=\frac{1}{2 D} \sum_{\sigma=1}^{D} \ln |\alpha|_{\sigma}=\ln y
$$

Так как $\alpha$ - вполне положительное число, то формула произведения также дает, что $\lambda(\alpha, \mathscr{D})=2(\ln x-\ln y), \ln x=h(\alpha)$. Тогда из предложения 5.5 следует, что

$$
\operatorname{sh}(\ln x-\ln y) \geqslant \exp (-\ln 2-\ln y)
$$

или после простых преобразований

$$
x^{2}-x-y^{2} \geqslant 0
$$

Решая это неравенство с учетом $x \geqslant 1$, получаем (5.4).

\section{§6. Формулировки основных результатов}

Напомним необходимые обозначения. Дано поле $\mathbb{K},[\mathbb{K}: \mathbb{Q}]=D, \mathscr{S} \subseteq\{1, \ldots, D\}$, $|\mathscr{S}|=S=s_{1}+2 s_{2}>0, s=s_{1}+s_{2}, \theta>0$. Набор $\mathscr{S}$ определяет расширенное логарифмическое пространство $\mathscr{E}(\mathscr{S})$ с нормами $H_{\theta}(\cdot, \mathscr{S}), H_{\theta}^{*}(\cdot, \mathscr{S})$. В $\mathscr{E}(\mathscr{S})$ имеется решетка $\Lambda(\mathscr{S})$, порождаемая $\mathbb{K}^{*}, \mathscr{A}$ - эпиморфизм $\Lambda(\mathscr{S})$ на $\mathbb{K}^{*}$. Положим для краткости

$$
\delta=\frac{D}{S}, \quad C=\frac{1+4 \theta}{2 \theta} .
$$

ТЕОРема 6.1. 1) Пусть $\mathbf{v}_{1}, \ldots, \mathbf{v}_{n} \in \Lambda(\mathscr{S})$ - линейно независимые векторь, $V_{k}=H_{\theta}\left(\mathbf{v}_{k}, \mathscr{S}\right), 1 \leqslant k \leqslant n$, тогда при $S<2 \pi C$ выполняется

$$
V_{1} \cdots V_{n} C^{n} n^{n} \delta \ln (4.64 \delta)>1
$$

2) Если числа $\beta_{k}=\mathscr{A}\left(\mathbf{v}_{k}\right) \in \mathbb{K}^{*}, k=1, \ldots, n$, мультипликативно независимьь, то условие $S<2 \pi C$ можнно отбросить.

\footnotetext{
${ }^{1}$ Когда статья находилась в печати, появилась работа [18], в которой доказьвается следствие 5.6.
} 
TЕОРема 6.2. Пусть $\mathbf{v}_{1}, \ldots, \mathbf{v}_{n} \in \Lambda(\mathscr{S})$ - линейно независимые вектоpвь, $V_{k}=H_{\theta}^{*}\left(\mathbf{v}_{k}, \mathscr{S}\right), 1 \leqslant k \leqslant n$. Положим $\Lambda_{n}=\Lambda \cap\left\langle\mathbf{v}_{1}, \ldots, \mathbf{v}_{n}\right\rangle_{\mathbb{R}}, \Lambda_{n}^{\prime}=$ $\left\langle\mathbf{v}_{1}, \ldots, \mathbf{v}_{n}\right\rangle_{\mathbb{Z}} \cdot$ Тогда

$$
V_{1} \cdots V_{n} C^{n}\left(e^{n /(2 \theta C)} n\right)^{S} \max \{6, n-1, \delta \ln (4.64 \delta)\}>\left[\Lambda_{n}: \Lambda_{n}^{\prime}\right] .
$$

СлеДСТВИЕ 6.3. 1) Пусть $\alpha_{1}, \ldots, \alpha_{n} \in \mathbb{K}^{*}-$ мультипликативно независимые числа, $H_{*}(\cdot)$ - из (4.3). Тогда при $\mathbb{K} \subseteq \mathbb{C}, \mathbb{K} \nsubseteq \mathbb{R}$

$$
H_{*}\left(\alpha_{1}\right) \cdots H_{*}\left(\alpha_{n}\right) 2.5^{n} e^{0.4 n} n^{2} \delta \ln (4.64 \delta)>1 .
$$

2) $E c л u \mathbb{K} \subseteq \mathbb{R}$, mo

$$
h\left(\alpha_{1}\right) \cdots h\left(\alpha_{n}\right) 2.5^{n} e^{0.2 n} n D^{n+1} \ln (4.64 D)>1 .
$$

ПРимечАния. 1. Главной целью работы является получение зависимости от $\delta$ вида (6.2), не меняющейся с ростом $n$. Главное в теореме 6.2 - это исключение из оценки множителя $n^{n}$ (ср. с (6.2)). При $n=1$ вместо (6.3) лучше взять (6.2).

2. Условие типа $S<2 \pi C$ необходимо, поскольку в $\Lambda(\mathscr{S})$ содержатся $\mathscr{A}$-прообразы единицы, имеющие одну координату (или пару) равную $2 \pi$. Так что у нас есть $s$ линейно независимых векторов, $s_{1}$ из которых имеют норму $2 \pi / S$ и $s_{2}-$ норму $4 \pi / S$. При большом $S$ оценка (6.2) может не выполняться. Отметим в связи с этим, что в (5.2) приводится $H_{\theta}(\alpha, \mathscr{S}), \alpha \neq 1$, а не $H_{\theta}(\mathbf{v}, \mathscr{S}), \mathbf{v} \neq \mathbf{0}$, так как в последнем случае (5.3) может не выполняться по той же причине.

3. Условие линейной независимости векторов $\mathbf{v}_{1}, \ldots, \mathbf{v}_{n}$ и неравенство $S<2 \pi C$ в теореме 6.1 можно заменить на более слабое требование, а именно: целочисленные линейные комбинации векторов $\mathbf{v}_{1}, \ldots, \mathbf{v}_{n}$ c коэффициентами, по модулю не превосходящими соответственно $1 /\left(C n V_{k}\right)$, не принадлежат $\operatorname{Ker} \mathscr{A}$. Аналогично можно уточнить теорему 6.2.

4. Отметим, что при $n=r$ оценка (6.5) не следует из степенной по $D$ оценки регулятора, поскольку в теореме Минковского неравенство (1.1) содержит в знаменателе слева $n$ !.

5. Оценки (6.4), (6.5) не следуют из предложения 5.1 и гипотезы Лемера, поскольку константа Лемера $\theta_{10}$ слишком мала, чтобы давать оценки при $n \geqslant 2$, аналогичные (6.4), (6.5).

6. Множитель $\left[\Lambda_{n}: \Lambda_{n}^{\prime}\right]$ в (6.3) превращает полученную оценку в аналог теоремы Минковского о последовательном минимуме (1.1). Этот множитель можно добавить и в неравенства $(6.2),(6.4),(6.5)$, тогда вместо $\delta \ln (4.64 \delta)$ в левой части появится множитель $\max \{6, n-1, \delta \ln (4.64 \delta)\}$, как в $(6.3)$.

\section{§7. Интерполяционный определитель}

Основой схемы Лорана [18] является следующее утверждение.

ПРЕДЛОЖЕНИЕ 7.1. Пусть данъ аналитические в нуле функции $f_{j}(\zeta)$, $1 \leqslant j \leqslant J$, и комплексные числа $z_{n}, 0 \leqslant n \leqslant J-1$. Тогда определитель $\Delta(\zeta)=\operatorname{det}\left(f_{j}\left(z_{n} \zeta\right)\right)_{j=1, \ldots, J}^{n=0, \ldots, J-1}$ имеет при $\zeta=0$ нуль кратности не меньие чем $J(J-1) / 2$.

Выделим фрагмент доказательства теорем, используюший схему Лорана, в отдельную лемму. 
Лемма 7.2. Пусть даны $\mathbf{x}_{j} \in \mathscr{E}(\mathscr{S}), 1 \leqslant j \leqslant J \quad(J \geqslant 2)$, сравнимые по $\bmod (\Lambda(\mathscr{S}))$, но не сравнимые по $\bmod (\operatorname{Ker} \mathscr{A})$, удовлетворяющие условиям:

$$
\begin{array}{ll}
\max _{j}\left|x_{j \sigma}\right| \leqslant X_{\sigma}, \quad \sigma \notin \mathscr{S} ; & \frac{1}{2 S} \sum_{\sigma \notin \mathscr{S}} X_{\sigma} \leqslant X ; \\
\max _{j}\left|z_{j \sigma}\right| \leqslant Y_{\sigma}, \quad \sigma \in \mathscr{S} ; & \frac{1}{S} \sum_{\sigma \in \mathscr{S}} Y_{\sigma} \leqslant Y,
\end{array}
$$

əде $X, X_{\sigma}, Y, Y_{\sigma}$ - некоторье числа.

Тогда выполняется неравенство

$$
\frac{D}{S} \frac{\ln J}{J-1}+1+\frac{X J}{J-1}-\ln \frac{2(J-1)}{J Y} \geqslant 0 .
$$

Содержательный смысл неравенства (7.3) состоит в том, что количество $J$ чисел малой высоты (если $X$ мало), близких к единице (если $Y$ мало), ограничено. Правда, вьписать решение этого неравенства в виде формулы непросто, поэтому оно оставлено пока в таком виде.

ДокАЗАТЕЛЬСТво. Пусть $\mathbf{x}_{j}=\mathbf{w}+\mathbf{w}_{j}, \mathbf{w}_{j} \in \Lambda(\mathscr{S}), \alpha_{j}=\mathscr{A}\left(\mathbf{w}_{j}\right)$. Положим $T=(J-1) / 2$, возьмем $\sigma \in \mathscr{S}$ и рассмотрим определители

$$
\begin{aligned}
\Delta_{\sigma}(\zeta) & =\zeta^{-2 J T} \operatorname{det}^{2}\left(\left(\alpha_{j}^{(\sigma)}\right)^{(n-T) \zeta}\right)_{j=1, \ldots, J}^{n=0, \ldots, J-1} \\
& =\zeta^{-2 J T} \operatorname{det}^{2}\left(e^{w_{j \sigma}(n-T) \zeta}\right)_{j=1, \ldots, J}^{n=0, \ldots, J-1} \in \mathbb{C}(\zeta), \quad \sigma \in \mathscr{S} .
\end{aligned}
$$

Очевидно, все $\Delta_{\sigma}(1)$ являются алгебраически сопряженньми для числа

$$
\Delta=\operatorname{det}^{2}\left(\alpha_{j}^{n-T}\right)_{j=1, \ldots, J}^{n=0, \ldots, J-1} \in \mathbb{K}
$$

Определим также функцию

$$
F(\zeta)=\prod_{\sigma \notin \mathscr{S}}|\Delta|_{\sigma} \cdot \prod_{\sigma \in \mathscr{S}} \Delta_{\sigma}(\zeta)
$$

(где только конечное число сомножителей отлично от единицы). Так как точки $\mathbf{w}_{j}$ не сравнимы по $\bmod (\operatorname{Ker} \mathscr{A})$, то числа $\alpha_{j}$ различны, и поэтому $\Delta \neq 0$. Тогда по формуле произведения имеем $|F(1)|=1$. Кроме того, по лемме 7.1 функция $F(\zeta)$ имеет устранимую особенность в нуле.

Теперь оценим $|F(\zeta)|$ сверху. Легко видеть, что

$$
\sum_{n=0}^{J-1}(n-T)=0, \quad \sum_{n=0}^{J-1}|n-T| \leqslant \frac{J^{2}}{4}
$$


Тогда, полагая $\gamma_{\sigma}=J^{J}$ для архимедовых нормирований и $\gamma_{\sigma}=1$ для неархимедовых, используя неравенство Адамара и (7.1), (7.2), (7.4), получаем с учетом равенства $w_{j \sigma}=z_{j \sigma}-w_{\sigma}$, что

$$
\begin{gathered}
|\Delta|_{\sigma} \leqslant \gamma_{\sigma} \exp \left(2 X_{\sigma} \sum_{j=1}^{J}|n-T|\right) \leqslant \gamma_{\sigma} \exp \left(\frac{1}{2} J^{2} X_{\sigma}\right), \quad \sigma \notin \mathscr{S}, \\
\left|\Delta_{\sigma}(\zeta)\right| \leqslant|\zeta|^{-2 J T} \gamma_{\sigma} \exp \left(\frac{1}{2} J^{2} Y_{\sigma}|\zeta|\right), \quad \sigma \in \mathscr{S} .
\end{gathered}
$$

Перемножая все $|\Delta|_{\sigma}$ и $\left|\Delta_{\sigma}(\zeta)\right|$ и логарифммируя, приходим к оценке

$$
\ln |F(\zeta)| \leqslant D J \ln J+X S J^{2}+\frac{1}{2} Y S|\zeta| J^{2}-2 J T S \ln |\zeta| .
$$

Если $4 T /(Y J) \leqslant 1$, то неравенство (7.3) очевидно. Если $4 T /(Y J)>1$, то, применяя принцип максимума к окружности $|\zeta|=4 T /(Y J)>1$, имеем

$$
0 \leqslant \ln |F(1)| \leqslant \frac{1}{2} D J \ln J+\frac{1}{2} X S J^{2}+J T S-J T S \ln \frac{4 T}{Y J},
$$

откуда после деления на $J S T=J S(J-1) / 2$ тоже получаем (7.3).

\section{§8. Доказательство теоремы 6.1}

Доказательство основано на том, что в предположении малости $V_{1}, \ldots, V_{n}$ удается найти достаточно много точек $\mathbf{x}_{j}$, чтобы получить противоречие с (7.3). Основная трудность заключается в решении этого неравенства. Оно будет решено приближенно для больших $J$, а затем полученная формула будет проверена для конечного числа сравнительно малых $J$.

Положим с учетом (6.1)

$$
\begin{gathered}
\eta=\frac{1}{2 \theta}, \quad C_{1}=n C=n(2+\eta), \\
J_{k}=\left[\frac{1}{C_{1} V_{k}}\right]+1, \quad T_{k}=\frac{J_{k}-1}{2} \quad(1 \leqslant k \leqslant n), \\
J=J_{1} \cdots J_{n}, \quad X=\frac{\eta}{C}, \quad Y=\frac{1}{2 C}
\end{gathered}
$$

пронумеруем наборы $\left\{j_{1}, \ldots, j_{n}\right\}, 0 \leqslant j_{k}<J_{k}, 1 \leqslant k \leqslant n$, индексами $j=1, \ldots, J$ и рассмотрим векторы

$$
\mathbf{x}_{j}=\left(j_{1}-T_{1}\right) \mathbf{v}_{1}+\cdots+\left(j_{n}-T_{n}\right) \mathbf{v}_{n} \in \mathscr{E}(\mathscr{S}), \quad j=1, \ldots, J .
$$

Очевидно, они сравнимы по $\bmod (\Lambda(\mathscr{S}))$. Проверим выполнение условия (7.1). С учетом $(8.1)$ и определения $H_{\theta}(\cdot, \mathscr{S})$ имеем

$$
\begin{aligned}
\frac{1}{2 S} \sum_{\sigma \notin \mathscr{S}} \max _{j}\left|x_{j \sigma}\right| & \leqslant \frac{1}{2 S} \sum_{\sigma \notin \mathscr{S}} \sum_{k=1}^{n}\left|v_{k \sigma}\right| \max _{j}\left|j_{k}-T_{k}\right| \leqslant \sum_{k=1}^{n} \mu\left(\mathbf{v}_{k}, \mathscr{S}\right) T_{k} \\
& \leqslant \sum_{k=1}^{n} \frac{\mu\left(\mathbf{v}_{k}, \mathscr{S}\right)}{2 C_{1} V_{k}} \leqslant \frac{1}{2 \theta C}=\frac{\eta}{2+\eta}=X .
\end{aligned}
$$


Аналогично проверяется условие (7.2). В частности, с учетом неравенства $S<2 \pi C$ получаем $\lambda\left(\mathbf{x}_{j}-\mathbf{x}_{l}, \mathscr{S}\right) \leqslant 2 Y<2 \pi / S$, поэтому векторы $\mathbf{x}_{j}$ не сравнимы по $\bmod (\operatorname{Ker} \mathscr{A})$. Теперь можно применить лемму 7.2. Перепишем $(7.3)$ в виде

$$
\delta \frac{\ln J}{J-1}+1+\frac{J}{J-1} \frac{\eta}{2+\eta}-\ln \frac{8(1+\eta / 2)(J-1)}{J} \geqslant 0 .
$$

Если мы покажем, что можно считать

$$
J<\xi(\delta)=\delta \ln (4.64 \delta),
$$

то ввиду условия $J>1 /\left(C_{1}^{n} V_{1} \cdots V_{n}\right)$ получим (6.2).

Пусть пока $J>6$. Положим для кратности $\varepsilon=1 /(J-1)$. Дифференцированием по $\eta$ получаем, что худшее значение $\eta$ в (8.3) равно $2 \varepsilon$. Худшее значение $\delta$ в случае невыполнения (8.4) определяется равенством $J=\xi(\delta)$. Тогда $(8.3)$ можно переписать в виде

$$
\delta \frac{\ln J}{J}+1-\frac{\ln 8}{1+\varepsilon} \geqslant 0, \quad J=\xi(\delta) .
$$

При $6<J \leqslant 20000$ неравенство проверяется компьютерным перебором: при каждом $J$ вычисляем $\varepsilon, \delta$ и подставляем их в (8.5). Программа на языке Qbasic требует для этого на компьютере Pentium несколько секунд.

При $J>20000$, положив $y=\ln (4.64 \delta) / 4.64$, получим

$$
\delta \frac{\ln J}{J}=1+\frac{\ln y}{4.64 y} \leqslant 1+\frac{1}{4.64 e}<\frac{\ln 8}{1.00005}-1 .
$$

Это противоречит (8.3) и поэтому (8.4) выполнено. (Именно для этого неравенства взята константа 4.64.)

Пусть теперь $J \leqslant 6$. Мы можем считать при $n \geqslant 2$ и при всех $k$, что $C_{1} V_{k}<1 / 2$, иначе, если $C_{1} V_{n} \geqslant 1 / 2$, то

$$
\begin{aligned}
C^{n} n^{n} V_{1} \cdots V_{n} & \geqslant \frac{1}{2} C^{n-1}(n-1)^{n-1} V_{1} \cdots V_{n-1}\left(1+\frac{1}{n-1}\right)^{n-1} \\
& \geqslant C^{n-1}(n-1)^{n-1} V_{1} \cdots V_{n-1}
\end{aligned}
$$

и мы можем действовать по индукции.

При $C_{1} V_{k}<1 / 2$ имеем $J_{k} \geqslant 3$, поэтому при $J \leqslant 6$ возможен лишш случай $n=1$. Здесь (6.2) несколько сильнее, чем (5.3), поскольку под знаком логарифма стоит 4.64, а не 4.8, но доказательство дословно совпадает с доказательством в [17], где константа 4.8 была выбрана из-за того, что проверка проводилась только до $J=1000$.

В случае мультипликативно независимых чисел $\beta_{k}$ нам не надо проверять различие чисел $\alpha_{j}$. Теорема 6.1 полностью доказана.

\section{§9. Доказательство теоремы 6.2}

Пусть $\mathscr{Z}=\left\{\mathbf{z}_{\sigma} \in \mathbb{C}^{n}: 1 \leqslant \sigma \leqslant S\right\}$, причем комплексные $\mathbf{z}_{\sigma}$ входят вместе с комплексно сопряженными; $\varepsilon>0$. Определим множество

$$
\mathscr{R}(\varepsilon ; \mathscr{Z})=\left\{\mathbf{x} \in \mathbb{R}^{n}:\left|\mathbf{x} \cdot \mathbf{z}_{\sigma}\right| \leqslant \varepsilon, 1 \leqslant \sigma \leqslant S\right\} .
$$


ЛЕмма 9.1. Пусть $\mathscr{W} \subseteq \mathbb{R}^{n}$ - выпуклое 0-симметричное тело, $\varepsilon>0$, $\mathbf{z}_{1}, \ldots, \mathbf{z}_{S} \in \mathbb{C}^{n}$. Положим

$$
\begin{gathered}
\varepsilon_{1}=\varepsilon_{1}(\mathscr{W}, \mathscr{Z})=\sup \left\{\left|\mathbf{x} \cdot \mathbf{z}_{\sigma}\right|: \mathbf{x} \in \mathscr{W}, 1 \leqslant \sigma \leqslant S\right\}, \\
\rho=\operatorname{rank}_{\mathbb{C}}\left\{\mathbf{z}_{1}, \ldots, \mathbf{z}_{S}\right\}, \quad c=\max \left\{1, \frac{\varepsilon_{1}}{\varepsilon}\right\} .
\end{gathered}
$$

Тогда

$$
\operatorname{Vol}_{n}(\mathscr{W} \cap \mathscr{R}(\varepsilon, \mathscr{Z})) \geqslant \operatorname{Vol}_{n}(\mathscr{W}) c^{-\rho}
$$

ДокАЗАТЕЛЬСтво. Если $c=1$ или $\rho=0$, то утверждение леммы очевидно, поскольку $\mathscr{W} \subseteq \mathscr{R}(\varepsilon, \mathscr{Z})$.

Пусть теперь $c>1, \rho>0$. Рассмотрим пространства

$$
\mathscr{X}=\langle\operatorname{Re} \mathscr{Z}, \operatorname{Im} \mathscr{Z}\rangle_{\mathbb{R}} \subseteq \mathbb{R}^{n}, \quad \mathscr{Y}=\mathscr{X}^{\perp} \subseteq \mathbb{R}^{n}
$$

Имеем $\operatorname{dim} \mathscr{X}=\rho, \operatorname{dim} \mathscr{Y}=n-\rho$. Рассмотрим функцию

$$
f(\mathbf{x})=\operatorname{Vol}_{n-\rho}(\mathscr{W} \cap(\mathbf{x}+\mathscr{Y})), \quad \mathbf{x} \in \mathscr{X} .
$$

Из теоремы Брунна-Минковского о выпуклом теле с учетом симметрии $\mathscr{W}$ следует, что для любого $\mathbf{x} \in \mathscr{X}$ функция $f(t \mathbf{x})$ убывает при $t \geqslant 0$. Пусть $\mathscr{R}^{\prime}-$ ортогональная проекция $\mathscr{R}(\varepsilon, \mathscr{Z})$ на $\mathscr{X}, \mathscr{W}^{\prime}-$ проекция $\mathscr{W}$ на $\mathscr{X}$, тогда ввиду того, что $\mathscr{W}^{\prime} \subseteq c \mathscr{R}^{\prime}$, получаем

$$
\begin{aligned}
\operatorname{Vol}_{n}(\mathscr{W} \cap \mathscr{R}(\varepsilon, \mathscr{Z})) & =\int_{\mathbf{x} \in \mathscr{R}^{\prime}} f(\mathbf{x}) d \operatorname{Vol}_{\rho}(\mathbf{x})=\int_{c \mathbf{x} \in c \mathscr{R}^{\prime}} f(\mathbf{x}) d \operatorname{Vol}_{\rho}(\mathbf{x}) \\
& \geqslant \int_{\mathbf{y} \in \mathscr{W}^{\prime}} f\left(\frac{\mathbf{y}}{c}\right) d \operatorname{Vol}_{\rho}\left(\frac{\mathbf{y}}{c}\right) \\
& \geqslant c^{-\rho} \int_{\mathbf{y} \in \mathscr{W}^{\prime}} f(\mathbf{y}) d \operatorname{Vol}_{\rho}(\mathbf{y})=c^{-\rho} \operatorname{Vol}_{n}\left(\mathscr{W}_{0}\right)
\end{aligned}
$$

что доказывает лемму.

Для доказательства теоремы 6.2 поступим следующим образом: вместо (8.1) с учетом (6.1) положим

$$
\eta=\frac{1}{2 \theta}, \quad C_{1}=C, \quad X=\frac{n \eta}{C}, \quad Y=\frac{1}{2 C e^{X}}, \quad Z=\frac{n}{2 C}
$$

определим векторы $\mathbf{z}_{\sigma}=\left(z_{1 \sigma}, \ldots, z_{n \sigma}\right), \sigma \in \mathscr{S}$, составленные из соответствующих координат векторов $\mathbf{v}_{j}$ из условий теоремы, и рассмотрим в пространстве векторов $\mathbf{w}=\left(w_{1}, \ldots, w_{n}\right) \in \mathbb{R}^{n}$ тела

$$
\mathscr{W}=\left\{\mathbf{w}:\left|w_{k}\right| \leqslant \frac{1}{2 C_{1} H_{\theta}^{*}\left(\mathbf{v}_{k}, \mathscr{S}\right)}, 1 \leqslant k \leqslant n\right\}, \quad \mathscr{W}_{0}=\mathscr{W} \cap \mathscr{R}(Y, \mathscr{Z}),
$$


а также линейный оператор $\mathscr{F}: \mathbb{R}^{n} \rightarrow \mathscr{E}$, задаваемый формулой

$$
\mathbf{x}=\mathscr{F}(\mathbf{w})=w_{1} \mathbf{v}_{1}+\cdots+w_{n} \mathbf{v}_{n}
$$

Очевидно, $\mathscr{F}$-прообразом $\Lambda_{n}^{\prime}=\left\langle\mathbf{v}_{1}, \ldots, \mathbf{v}_{n}\right\rangle_{\mathbb{Z}}$ является $\mathrm{M}_{n}^{\prime}=\mathbb{Z}^{n}$, и пусть $\mathrm{M}_{n}-$ прообраз $\Lambda_{n}$, при этом

$$
\operatorname{Vol}_{n}\left(\mathrm{M}_{n}\right)=\frac{\operatorname{Vol}_{n}\left(\mathrm{M}_{n}^{\prime}\right)}{\left[\mathrm{M}_{n}: \mathrm{M}_{n}^{\prime}\right]}=\frac{1}{\left[\Lambda_{n}: \Lambda_{n}^{\prime}\right]}
$$

По теореме Блихфельдта в теле $\mathscr{W}_{0}$ найдутся $J$ точек $\mathbf{w}_{1}, \ldots, \mathbf{w}_{j}$, сравнимых по $\bmod \left(\mathrm{M}_{n}\right)$

$$
J>\frac{\operatorname{Vol}_{n}\left(\mathscr{W}_{0}\right)}{\operatorname{Vol}_{n}\left(\mathrm{M}_{n}\right)}=\operatorname{Vol}_{n}\left(\mathscr{W}_{0}\right)\left[\Lambda_{n}: \Lambda_{n}^{\prime}\right]
$$

Положим $\mathbf{x}_{j}=\mathscr{F}\left(\mathbf{w}_{j}\right), j=1, \ldots, J$. Как и в случае теоремы 6.1 , для $\mathbf{x}_{j}$ выполнено условие (7.1) с $X$ из (9.1), а (7.2) выполнено по построению тела $\mathscr{W}_{0}$. Кроме того, $\varepsilon_{1}(\mathscr{W}, \mathscr{Z}) \leqslant Z=n /(2 C)$, поэтому по лемме 9.1 имеем

$$
\operatorname{Vol}_{n}\left(\mathscr{W}_{0}\right) \geqslant \operatorname{Vol}_{n}(\mathscr{W}) \max \left\{1, \frac{Z}{Y}\right\}^{-\rho} \geqslant \frac{1}{(Z / Y)^{S} C_{1}^{n} V_{1} \ldots V_{n}}
$$

Покажем, что

$$
J \leqslant \max \{6, n-1, \delta \ln (4.64 \delta)\} .
$$

Поскольку $\sigma$-координаты $(\sigma \in \mathscr{S})$ векторов $\mathbf{x}_{j}$ не превосходят по модулю $Y<\pi$, то $\mathbf{x}_{j}$ не сравнимы по $\bmod (\operatorname{Ker} \mathscr{A})$ и можно применить лемму 7.2 , что дает неравенство (7.3) с $X, Y$ из (9.1) , и (7.3) можно записать в виде

$$
\delta \frac{\ln J}{J-1}+1+\frac{J}{J-1} \frac{n \eta}{2+\eta}-\frac{n \eta}{2+\eta}-\ln \frac{8(1+\eta / 2)(J-1)}{J} \geqslant 0 .
$$

Если считать, что $J \geqslant n$, то

$$
\frac{n J}{J-1}-n \leqslant \frac{J}{J-1}
$$

и (9.4) можно заменить на (8.4). При $J>6$ из (8.4) следует (8.5), что доказывает (9.3) и теорему 6.2 .

ЗАмечАниЕ. Приведенный в доказательстве прием усечения множества $\mathscr{W}$, позволяющий устранить из оценки множитель $n^{n}$, был ранее использован автором при оценке линейных форм от логарифмов алгебраических чисел. 


\section{§10. Доказательство следствия 6.3}

$\Pi$ ри $\mathbb{K} \subseteq \mathbb{R}$ возьмем $\sigma$, соответствуюшее данному вложению, $\mathscr{S}=\{\sigma\}, S=1$, $\delta=D$. В комплексном случае возьмем пару соответствующих комплексно сопряженных $\sigma, \tau, \mathscr{S}=\{\sigma, \tau\}, S=2, \delta=D / 2$. Отметим, что здесь $\theta=1$, $H_{1}(\cdot, \mathscr{S})=H_{1}^{*}(\cdot, \mathscr{S})$.

Очевидно, в комплексном случае $H_{*}(\alpha, \mathscr{S}) \geqslant H_{1}(\alpha, \mathscr{S})$. В действительном случае с помощю замены $\alpha \rightarrow \pm \alpha^{ \pm 1}$ можно получить $\lambda(\alpha, \mathscr{S})=\ln \alpha>0$, откуда с учетом формулы произведения также имеем $D h(\alpha) \geqslant H_{1}(\alpha, \mathscr{S})$. Это позволяет применить теоремы 6.1 и 6.2. При $n=1$ оценки $(6.4),(6.5)$ следуют непосредственно из (6.2).

При $n \geqslant 2$ применим (6.3). Покажем, что максимум в (6.3) можно считать равным $\delta \ln (4.64 \delta)$. Сделаем некоторые упрощения. Мы можем отбросить $\alpha_{j}$, не явлющиеся алгебраическими единицами, поскольку для них

$$
e^{0.2} 2.5 \frac{H(\alpha)}{S} \geqslant e^{0.2} 2.5 \frac{\ln 2}{2}>1
$$

Значит, можно считать, что $2 \leqslant n \leqslant r<D$ и $D \ln (4.64 D)>6$. Тем самьм неравенство (6.5) доказано при $\mathbb{K} \subseteq \mathbb{R}$.

В комплексном случае при $D \geqslant 5$ также имеем $\delta \ln (4.64 \delta)>6$, и (6.4) следует из (6.3). Кроме того, здесь $D-2 \geqslant r \geqslant n \geqslant 2$, поэтому остается только случай $n=2, D=4$. Но $H(\alpha)>0.28$ (см. [12] и предложение 3.3) и (6.4) проверяется непосредственно.

\section{Список литературы}

1. Боревич З. И., Шафаревич И. Р. Теория чисел. М.: Наука, 1972.

2. Касселс Дж. Введение в геометрию чисел. М.: Мир, 1965.

3. Tsfasman M. A. Global fields, codes and sphere packings. Journees arithmetiques, Exp. Congr., Luminy/Fr. 1989 // Astérisque. 1991. № 198-200. P. 373-396.

4. Pohst M. E. Computational algebraic number theory. DMV Seminar. № 21. Basel: Birkhäuser, 1993.

5. Ленг C. Основы диофантовой геометрии. М.: Мир, 1986.

6. Lehmer D. H. Factorization of certain cyclotomic functions // Ann. of Math. 1993. V. 34. № 2. P. 461-479.

7. Dobrowolski E. On a question of Lehmer and the number of irreducible factors of a polynomial // Acta Arith. 1979. V. 34. P. 391-401.

8. Матвеев E. M. О связи меры Малера и дискриминанта алгебраических чисел // Матем. заметки. 1996. Т. 59. № 3. С. 415-420.

9. Blanksby P. E., Montgomery H. L. Algebraic integers near the unit circle // Acta Arith. 1971. V. 18. P. 355-369.

10. Smyth C.J. On the product of conjugates outside the unit circle of an algebraic integer // Bull. London Math. Soc. 1971. V. 3. P. 169-175.

11. Schinzel $A$. On the product of the conjugates outside the unit circle of an algebraic number // Acta Arith. 1973. V. 24. P. 385-399.

12. Boyd D. W. Reciprocal polynomials having small measure. I // Math. Comp. 1980. V. 35. № 152. P. 1361-1377; II // Math. Comp. 1989. V. 53. № 187. P. 355-357.

13. Zimmert R. Ideale kleiner Norm in Idealklassen und eine Regulatorabschätzung // Invent. Math. 1981. V. 62. P. 367-380.

14. Friedman E. Analytic formulas for the regulator of a number field // Invent. Math. 1989. V. 98. P. 599-622. 
15. Waldschmidt M. A lower bound for linear forms in logarithms // Acta Arith. 1980. V. 37. P. 257-283.

16. Матвеев E. М. Об одном типе мульипликативных алгебраических решеток // Тезисы докладов международной конференции "Современные проблемы теории чисел". Тула 20-25 сентября 1993 г. Тула: ТГПИ, 1993. С. 107.

17. Матвеев E. М. Об алгебраических числах малой логарифомической высоты // Диофантовы приближения. Сер. "Монографии и тематические сборники". Математические записки. 1996. Т. 2. С. 90-98.

18. Flammang $V$. Inegalites sur la mesure de Mahler d'un polynome // J. Théor. Nombres Bordeaux. 1997. V. 9. №1. P. 69-74.

19. Laurent $M$. Sur quelques résultats récent de trancsendance. Journees arithmetiques, Exp. Congr., Luminy/Fr. 1989 // Astérisque. 1991. № 198-200. P. 209-230.

Московская государственная текстильная

Поступила в редакцию академия им. А.Н. Косьгина 04.04.1997 и 10.03.1998 